\title{
THE CHANGE IN ELECTRIC POTENTIAL DUE TO LIGHTNING*
}

\author{
William W. Hager ${ }^{1}$ and Beyza Caliskan Aslan $^{2}$
}

\begin{abstract}
The change in the electric potential due to lightning is evaluated. The potential along the lightning channel is a constant which is the projection of the pre-flash potential along a piecewise harmonic eigenfunction which is constant along the lightning channel. The change in the potential outside the lightning channel is a harmonic function whose boundary conditions are expressed in terms of the pre-flash potential and the post-flash potential along the lightning channel. The expression for the lightning induced electric potential change is derived both for the continuous equations, and for a spatially discretized formulation of the continuous equations. The results for the continuous equations are based on the properties of the eigenvalues and eigenfunctions of the following generalized eigenproblem: Find $u \in H_{0}^{1}(\Omega), u \neq 0$, and $\lambda \in \mathbb{R}$ such that $\langle\nabla u, \nabla v\rangle_{\mathcal{L}}=\lambda\langle\nabla u, \nabla v\rangle_{\Omega}$ for all $v \in H_{0}^{1}(\Omega)$, where $\Omega \subset \mathbb{R}^{n}$ is a bounded domain (a box containing the thunderstorm), $\mathcal{L}$ is a subdomain (the lightning channel), and $\langle\cdot, \cdot\rangle_{\Omega}$ is the inner product $\langle\nabla u, \nabla v\rangle_{\Omega}=\int_{\Omega} \nabla u \cdot \nabla v \mathrm{~d} x$.
\end{abstract}

Mathematics Subject Classification. 35J25, 35Q60, 35A20, 35P10.

Received September 28, 2007. Revised March 16, 2008.

Published online July 4, 2008.

\section{INTRODUCTION}

During lightning, a region of space, the lightning channel, becomes highly conductive leading to an abrupt change in the electric potential throughout the atmosphere. If the conductivity along the lightning channel became infinite, then lightning would cause a jump discontinuity in the electric potential. In this paper, we evaluate the change in the electric potential that results from infinite conductivity along the lightning channel.

The following equations model the evolution of the electric potential in a domain $\Omega$, assuming the time derivative of the magnetic flux density can be neglected:

$$
\begin{aligned}
\Delta \frac{\partial \phi}{\partial t} & =-\nabla \cdot(\sigma \nabla \phi)+\nabla \cdot \mathbf{J} \quad \text { in } \Omega \times[0, \infty), \\
\phi(\mathbf{x}, t) & =0, \quad(\mathbf{x}, t) \in \partial \Omega \times[0, \infty), \\
\phi(\mathbf{x}, 0) & =\phi_{0}(\mathbf{x}), \quad \mathbf{x} \in \Omega,
\end{aligned}
$$

Keywords and phrases. Lightning, electric potential, Ampere's law, Maxwell's equations, Laplacian, generalized eigenproblem, double layer potential, complete eigenbasis.

* This material is based upon work supported by the National Science Foundation under Grants 0203270, 0619080, 0620286, 0724750 , and 0724771 .

${ }^{1}$ Department of Mathematics, University of Florida, PO Box 118105, 32611-8105 Gainesville, Florida, USA.

hager@math.ufl.edu; http://www.math.ufl.edu/ hager

2 Department of Mathematics and Statistics, University of North Florida, 32224 Jacksonville, Florida, USA. aslan@unf.edu; http://www.unf.edu/coas/math-stat/ aslan 


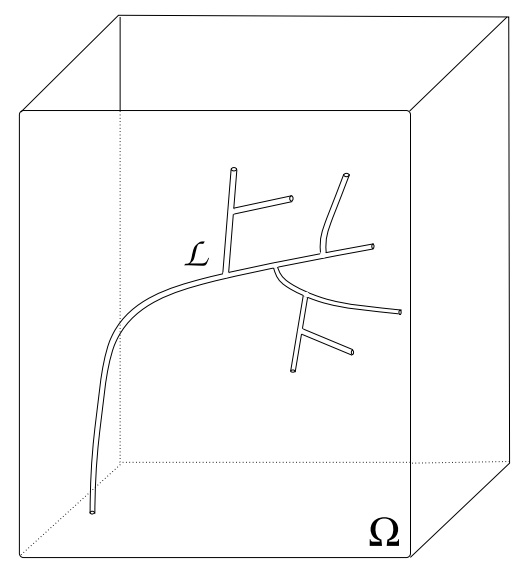

FiguRE 1. A sketch of $\mathcal{L}$ and $\Omega$ for a lightning discharge.

where $\Omega \subset \mathbb{R}^{n}$ is a bounded domain with boundary $\partial \Omega, \sigma \geq 0$ lies in the space $L^{\infty}(\Omega)$ of essentially bounded functions defined on $\Omega$, and the components of the forcing term $\mathbf{J}$ lie in $L^{2}(\Omega)$, the usual space of square integrable functions defined on $\Omega$. The initial condition $\phi_{0}$ is assumed to lie in $H_{0}^{1}(\Omega)$, the Sobolev space consisting of functions which vanish on $\partial \Omega$ and with first derivatives in $L^{2}(\Omega)$. Although Maxwell's equations describe the electromagnetic fields in $\mathbb{R}^{3}$, the mathematical analysis throughout this paper is developed in $\mathbb{R}^{n}$, where $n$ is an arbitrary positive integer.

Equation (1.1) is interpreted in a weak sense, in which case the divergence $\nabla \cdot \mathbf{J}$ represents a function in $H^{-1}(\Omega)$ when the components of $\mathbf{J}$ lie in $L^{2}(\Omega)$. For $f \in H^{-1}(\Omega)$, let $\Delta^{-1} f$ denote the weak solution $u$ to the problem

$$
\Delta u=f, \quad u=0 \text { on } \partial \Omega .
$$

Hence, $\Delta^{-1}: H^{-1}(\Omega) \rightarrow H_{0}^{1}(\Omega)$. After multiplying (1.1) by $\Delta^{-1}$, we obtain an equation of the form

$$
\frac{\partial \phi}{\partial t}=\mathcal{B} \phi+f, \quad \mathcal{B}=-\Delta^{-1}(\nabla \cdot(\sigma \nabla)), \quad f=\Delta^{-1}(\nabla \cdot \mathbf{J}) .
$$

Since $\mathcal{B}$ is a bounded, linear operator on $H_{0}^{1}(\Omega)$, it follows from [3], Corollary 2.2 .3 , that $\mathcal{B}$ is the infinitesimal generator for a strongly continuous semigroup on $H_{0}^{1}(\Omega)$. Since $f$ and $\phi_{0}$ lie in $H_{0}^{1}(\Omega)$, it follows from [1], Theorem 7.10, that equation (1.4), with the boundary conditions (1.2) and (1.3), has a unique solution $\phi \in$ $C^{1}\left([0, \infty) ; H_{0}^{1}(\Omega)\right)$.

In a thunderstorm, $\sigma$ is the conductivity divided by the permittivity of the atmosphere, $\Omega$ is any large domain extending from the Earth to the ionosphere which contains the thundercloud in its interior, and $\mathbf{J}$ is due to transport by wind of charged ice and water particles in the cloud. Although $\mathbf{J}$ is a function of time, we focus on the potential change during lightning, which we consider infinitely fast. Hence, during a lightning event, J is essentially time invariant. The potential of the ionosphere is not zero, however, we can make a change of variables to subtract off the "fair-field potential" (the potential of the atmosphere when the thundercloud is removed) and transform the problem to the form (1.1)-(1.3) where the potential vanishes on $\partial \Omega$.

A possible lightning channel is sketched in Figure 1. Mathematically, the lightning channel $\mathcal{L}$ could be any connected, open set contained in $\Omega$ with its complement $\mathcal{L}^{c}=\Omega \backslash \mathcal{L}$ connected. More realistically, we should view $\mathcal{L}$ as a connected network of thin open tubes. The case where $\mathcal{L}$ touches $\partial \Omega$, as would happen during a cloud-to-ground flash, is treated as a limit in which $\mathcal{L}$ approaches arbitrarily closely to the boundary of $\Omega$.

Let $\Psi$ be the characteristic function for $\mathcal{L}$ ( $\Psi$ is identically 0 except in $\mathcal{L}$ where $\Psi$ is 1 ). The effect of lightning is to ionize the domain $\mathcal{L}$, in essence, replacing $\sigma$ in (1.1) by $\sigma+\tau \Psi$ where $\tau$ is large. If the lightning occurs 
at $t=0$, then in the moments after the lightning, the electric potential is governed by the equation

$$
\frac{\partial \Delta \phi}{\partial t}=-\nabla \cdot(\sigma \nabla \phi)-\tau \nabla \cdot(\Psi \nabla \phi)+\nabla \cdot \mathbf{J} \quad \text { in } \Omega \times[0, \infty),
$$

subject to the boundary conditions (1.2) and (1.3). Here the scalar $\tau$ reflects the change in conductivity in the lightning channel. If $\phi_{\tau}(\mathbf{x}, t)$ denotes the solution to (1.5) at position $x \in \Omega$ and time $t$, and if the lightning is infinitely fast and the conductivity of the channel is infinitely large, then the potential right after the lightning is given by

$$
\phi^{+}(\mathbf{x})=\lim _{t \rightarrow 0^{+}} \lim _{\tau \rightarrow \infty} \phi_{\tau}(\mathbf{x}, t) .
$$

We show that $\phi^{+}$can be expressed in the following way:

Theorem 1.1. If $\partial \Omega$ is $C^{2}$ and $\partial \mathcal{L}$ is $C^{2, \alpha}$, for some $\alpha \in(0,1)$ (the exponent of Hölder continuity for the second derivative), then the electric potential $\phi^{+}$immediately after the lightning discharge is given by

$$
\phi^{+}(\mathbf{x})= \begin{cases}\phi_{L} & \text { if } \mathbf{x} \in \mathcal{L}, \\ \phi_{0}(\mathbf{x})+\xi(\mathbf{x}) & \text { if } \mathbf{x} \in \mathcal{L}^{c}\end{cases}
$$

where

$$
\phi_{L}=\frac{\left\langle\nabla \phi_{0}, \nabla \Pi\right\rangle_{\Omega}}{\langle\nabla \Pi, \nabla \Pi\rangle_{\Omega}}
$$

and where $\Pi$ and $\xi$ are harmonic functions in $\mathcal{L}^{c}$ with boundary conditions as specified below:

$$
\begin{aligned}
\Delta \Pi=0 \text { in } \mathcal{L}^{c}, \quad \Pi=0 \text { on } \partial \Omega, \quad \Pi=1 \text { in } \mathcal{L}, \\
\Delta \xi=0 \text { in } \mathcal{L}^{c}, \quad \xi=0 \text { on } \partial \Omega, \quad \xi=\phi_{L}-\phi_{0} \text { on } \partial \mathcal{L} .
\end{aligned}
$$

Here $\langle\cdot, \cdot\rangle_{\Omega}$ is the $L^{2}(\Omega)$ inner product

$$
\langle\nabla u, \nabla v\rangle_{\Omega}=\int_{\Omega} \nabla u \cdot \nabla v \mathrm{~d} x
$$

Thus $\phi^{+}$has the constant value $\phi_{L}$ along the lightning channel $\mathcal{L}$ and the change in the potential due to lightning has been expressed in terms of the potential $\phi_{0}$ before the lightning and the lightning channel $\mathcal{L}$. When $\mathcal{L}$ touches $\partial \Omega$, as it would during a cloud-to-ground flash, $\phi_{L}=0$ and $\Pi$ can be eliminated. That is, as $\mathcal{L}$ approaches the boundary of $\Omega, \Pi$ develops a jump singularity since $\Pi=1$ on $\mathcal{L}$ and $\Pi=0$ on $\partial \Omega$. Hence, $\nabla \Pi$ approaches a delta function as $\mathcal{L}$ approaches $\partial \Omega$. Since the delta function is squared in the denominator of $\phi_{L}$ while the numerator is finite, $\phi_{L}$ tends to 0 as $\mathcal{L}$ approaches $\partial \Omega$. Thus in a cloud-to-ground flash, the change $\xi$ in electric potential due to the lightning is the solution to (1.10) with $\phi_{L}=0$.

This formula for the potential change due to lightning can be exploited in a stepwise fashion to grow a lightning channel, as we show in $[4,6]$. In other words, the lightning channel starts out as a tiny line segment where the magnitude of the electric field first reaches the local breakdown threshold of the atmosphere. A new channel segment is oriented along the electric field lines and appended to the existing channel at a point on $\partial \mathcal{L}$ where the electric field magnitude is largest. The channel continues to grow in small steps until the magnitude of the electric field drops beneath the breakdown threshold everywhere on the boundary of $\mathcal{L}$. See $[4,6]$ for further discussion and simulations of channel growth. In this paper, we focus on the evaluation of the electric potential after a lightning discharge along a prescribed channel.

Lightning channels can be estimated using the time-of-arrival of VHF pulses generated during lightning [8, $9,12,13,15,17,18]$. These pulses can be used to form a graph, which we call the "pulse graph" of a flash [7], and which approximates the lightning channel. Ground or balloon-based electric field mills or antenna systems can be used to estimate $\mathbf{J}$ in (1.1) (see [6,7]). Hence, by numerically integrating (1.1)-(1.3), we can compute $\phi$, 
which gives us the pre-flash potential $\phi_{0}$ of Theorem 1.1. The potential change associated with lightning, given by Theorem 1.1, can be used to compute both lightning charge transport, using Gauss' law, and the energy $E$ associated with a lightning discharge using the formula

$$
E=\frac{\epsilon}{2} \int_{\Omega}\left(\left|\nabla \phi_{0}(\mathbf{x})\right|^{2}-\left|\nabla \phi^{+}(\mathbf{x})\right|^{2}\right) \mathrm{d} x .
$$

Previously, Marshall and Stolzenburg [10] (also see [14], Sect. 4.2) have estimated the energy associated with a lightning flash from balloon soundings of the electric field in a thunderstorm and assumed minimum and maximum values for the charge transfer. The formula (1.12) would provide a more precise way to estimate flash energy without additional assumptions.

In our previous analysis of the potential change due to lightning, the continuous equation (1.5) was discretized and we studied limits in the discrete equations. Discretizing the spatial domain in (1.5) leads to a linear ordinary differential equation:

$$
\mathbf{A} \dot{\boldsymbol{\Phi}}+\left(\mathbf{B}+\tau \mathbf{U}^{\top}\right) \boldsymbol{\Phi}+\mathbf{i}=\mathbf{0}, \quad \boldsymbol{\Phi}(0)=\boldsymbol{\Phi}_{0},
$$

where the dot denotes time derivative, $\mathbf{A}$ and $\mathbf{B}$ are $n$ by $n$ symmetric, positive definite matrices, $\mathbf{U}$ is an $n$ by $m$ matrix with each column completely zero except for a +1 and -1 entry, and $\mathbf{i} \in \mathbb{R}^{n}$ (see [5]). For each column of $\mathbf{U}$, the location of the +1 and -1 correspond to an arc in the lightning channel connecting two points in the mesh associated with the spatial discretization. Let $\boldsymbol{\Phi}_{\tau}(t)$ denote the solution to (1.13) at time $t$. The following limit is established for the discrete potential right after lightning at $t=0$ :

Theorem 1.2. If $\mathbf{A}$ and $\mathbf{B}$ are $n$ by $n$ symmetric matrices, $\mathbf{A}$ is positive definite, and $\mathbf{U}$ is an $n$ by $m m a t r i x$ of rank $m$, then we have

$$
\boldsymbol{\Phi}^{+}=\lim _{t \rightarrow 0^{+}} \lim _{\tau \rightarrow \infty} \boldsymbol{\Phi}_{\tau}(t)=\boldsymbol{\Phi}_{0}-\mathbf{A}^{-1} \mathbf{U}\left(\mathbf{U}^{\top} \mathbf{A}^{-1} \mathbf{U}\right)^{-1} \mathbf{U}^{\top} \boldsymbol{\Phi}_{0}
$$

In [5] this formula was obtained when the time derivative in (1.13) was approximated by an implicit Euler scheme. That is, the potential $\boldsymbol{\Phi}(\Delta t)$ was approximated by the solution $\boldsymbol{\Phi}^{1}$ to the following equation, which corresponds to an implicit Euler discretization of (1.13):

$$
\mathbf{A}\left(\boldsymbol{\Phi}^{1}-\boldsymbol{\Phi}_{0}\right)+\Delta t\left(\mathbf{B}+\tau \mathbf{U}^{\top}\right) \boldsymbol{\Phi}^{1}+\Delta t \mathbf{i}=\mathbf{0} .
$$

We observed in [5] that as $\tau$ tends to $\infty$ and as $\Delta t$ tends to $0, \boldsymbol{\Phi}^{1}$ approaches the limit (1.14).

In [4] we examined the continuous differential equation (1.13) without discretizing time. We showed that the formula (1.14) remains valid provided $\mathbf{A}$ and $\mathbf{B}$ commute (that is, they share a common set of eigenvectors [16], p. 249). Now we will establish (1.14) in the more general setting where $\mathbf{A}$ is symmetric, positive definite and $\mathbf{B}$ is symmetric. Thus (1.14) is a rather general discrete analogue of (1.7).

By (1.14), $\mathbf{U}^{\top} \boldsymbol{\Phi}^{+}=\mathbf{0}$. Hence, in the lightning context, where each column of $\mathbf{U}$ is zero except for a single +1 and $-1, \mathbf{U}^{\top} \boldsymbol{\Phi}^{+}=\mathbf{0}$ is equivalent to the equality of components of $\boldsymbol{\Phi}^{+}$corresponding to the location of the +1 and -1 in each column of $\mathbf{U}$. Analogously, for the continuous equation (1.5), $\phi^{+}$in the domain $\mathcal{L}$ is the projection of $\phi_{0}$ on $\Pi$, where $\Pi$ is constant on $\mathcal{L}$.

In the lightning context, the matrix $\mathbf{A}$ in the differential equation (1.13) is a discretization of the Laplacian. In (1.14), $\mathbf{A}^{-1}$ appears twice, in the capacitance matrix factor $\mathbf{U}^{\top} \mathbf{A}^{-1} \mathbf{U}$ as well as in the external factor $\mathbf{A}^{-1}$. Analogously, in (1.9) and (1.10) we solve a harmonic equation twice, once to obtain $\Pi$, and then again when we compute $\xi$. The boundary condition for $\xi$ on $\partial \mathcal{L}$ connects the potential $\phi_{0}$ before the lightning with the potential $\phi_{L}$ along the lightning channel.

The potential change in the discrete context is $\boldsymbol{\Phi}^{+}-\boldsymbol{\Phi}_{0}$. Let $\mathbf{V}$ be an $n$ by $n-m$ matrix of rank $n-m$ whose columns are orthogonal to the columns of $\mathbf{U}$. By (1.14) we have $\mathbf{V}^{\top} \mathbf{A}\left(\boldsymbol{\Phi}^{+}-\boldsymbol{\Phi}_{0}\right)=\mathbf{0}$, which is the discrete analogue of the result (1.10) in Theorem 1.1 that the potential change $\xi$ is harmonic in $\mathcal{L}^{c}$. 
The evaluation of the limit (1.7) was motivated by the techniques developed to obtain the discrete limit (1.14). Even though the matrices $\mathbf{A}, \mathbf{B}$, and $\mathbf{U}^{\top} \mathbf{U}^{\top}$ in (1.13) are all symmetric, the solution $\mathbf{\Phi}$ to the differential equation is not symmetric. The key step in our general derivation of (1.14) involves decomposing the solution into a product of symmetric factors where we could exploit symmetry, and could analyze how $\tau$ effects eigenvalues. For the continuous equation (1.5), the analogue of symmetry is achieved by reformulating the equation in terms of the eigenfunctions of the following generalized eigenproblem: Find $u \in H_{0}^{1}(\Omega), u \neq 0$, and $\lambda \in \mathbb{R}$ such that

$$
\langle\nabla u, \nabla v\rangle_{\mathcal{L}}=\lambda\langle\nabla u, \nabla v\rangle_{\Omega}
$$

for all $v \in H_{0}^{1}(\Omega)$. Throughout the paper, we view $H_{0}^{1}(\Omega)$ as a Hilbert space for which the inner product between functions $u$ and $v \in H_{0}^{1}(\Omega)$ is given by (1.11). In [2] we prove the following result which implies that the eigenfunctions associated with (1.15) form a complete orthonormal set:

Theorem 1.3. If $\partial \Omega$ is $C^{2}$ and $\partial \mathcal{L}$ is $C^{2, \alpha}$, for some $\alpha \in(0,1)$, then any $f \in H_{0}^{1}(\Omega)$ has an expansion of the form

$$
f=\sum_{i=1}^{\infty} c_{i} \phi_{i}
$$

where the $\phi_{i}$ are orthonormal eigenfunctions of (1.15) relative to the inner product (1.11), and $c_{i}=\left\langle\nabla f, \nabla \phi_{i}\right\rangle$. Here the convergence is with respect to the norm of $H_{0}^{1}(\Omega)$.

The analysis in [2] exhibits four classes of eigenfunctions for (1.15):

(1) The function $\Pi$ which is 1 on $\mathcal{L}$ and harmonic on $\mathcal{L}^{c}$; the eigenvalue is 0 .

(2) Functions in $H_{0}^{1}(\Omega)$ with support in $\mathcal{L}^{c}$; the eigenvalue is 0.

(3) Functions in $H_{0}^{1}(\Omega)$ with support in $\mathcal{L}$; the eigenvalue is 1.

(4) Excluding $\Pi$, the harmonic extensions into $\mathcal{L}$ and $\mathcal{L}^{c}$ of the eigenfunctions of a double layer potential on $\partial \mathcal{L}$. The eigenvalues are contained in the open interval $(0,1)$. The only possible accumulation point is $\lambda=1 / 2$.

The eigenfunction $\Pi$ is particularly important. According to Theorem 1.1, the potential along the lightning channel is the projection of the pre-flash potential $\phi_{0}$ along the eigenfunction $\Pi$.

The paper is organized as follows: In Section 2 we explain how (1.1) is obtained from Maxwell's equation. In Section 3 we establish the discrete limit (1.14) in a general setting. In Section 4 we use the eigendecomposition given in Theorem 1.3 to reformulate the continuous equations (1.5). In Section 5 we evaluate the limit (1.7). A closed form solution for the limit in 1-dimension is given in Section 6. Final conclusions appear in Section 7.

Notation. Throughout the paper, we use the following notation. $\mathbb{N}$ is the set of positive integers (natural numbers) and $\ell^{2}$ is the space of square summable sequences: $\mathbf{x} \in \ell^{2}$ if

$$
\sum_{i=1}^{\infty} x_{i}^{2}<\infty .
$$

If $\mathbf{x} \in \mathbb{R}^{n}$ or $\mathbf{x} \in \ell^{2}$, then $\|\mathbf{x}\|$ denotes the Euclidean norm. If $\mathbf{M}$ is a matrix, then $\|\mathbf{M}\|$ is the 2-norm defined by

$$
\|\mathbf{M}\|=\sup \{\|\mathbf{M x}\|:\|\mathbf{x}\|=1\}=\sup _{\|\mathbf{x}\|=1} \sup _{\|\mathbf{y}\|=1} \mathbf{y}^{\top} \mathbf{M} \mathbf{x} .
$$

When $\mathbf{M}$ is finite dimensional, $\|\mathbf{M}\|$ is its largest singular value. The complement of $\mathcal{L}$ is $\mathcal{L}^{c}=\Omega \backslash \mathcal{L}$.

We let $\mathbf{I}$ and $\mathbf{0}$ denote the $n$ by $n$ identity and zero matrices respectively. In general, $\mathbf{I}_{m \times m}$ and $\mathbf{0}_{m \times n}$ denote $m$ by $m$ and $m$ by $n$ versions of the identity and the zero matrices.

$C_{0}^{\infty}(\Omega)$ is the collection of infinitely differentiable functions with compact support in $\Omega$ and $H_{0}^{1}(\Omega)$ is the closure of $C_{0}^{\infty}(\Omega)$ in $H^{1}(\Omega)$. $C^{k}$ denotes the set of $k$-times continuously differentiable functions, while $C^{k, \alpha}$ is the subset of $C^{k}$ whose $k$ th derivative is Hölder continuous with exponent $\alpha$. 


\section{MAXWELL'S EQUATIONS}

Equations (1.1) or (1.5) are derived from Maxwell's equations for linear materials. By Ampere's law, we have

$$
\operatorname{curl}(\mathbf{H})=\mathbf{J}_{T}+\epsilon \frac{\partial \mathbf{E}}{\partial t},
$$

where $\mathbf{H}$ is the magnetic field, $\mathbf{J}_{T}$ is the total current density, $\mathbf{E}$ is the electric field, and $\epsilon$ is the permittivity of air. $\mathbf{J}_{T}$ is partly due to the movement of charged ice and water particles in the cloud and partly due to the electrical conductivity in the cloud: $\mathbf{J}_{T}=\mathbf{J}_{p}+\sigma_{a} \mathbf{E}$, where $\sigma_{a}$ is the conductivity of the atmosphere. Hence,

$$
\operatorname{curl}(\mathbf{H})=\epsilon \frac{\partial \mathbf{E}}{\partial t}+\sigma_{a} \mathbf{E}+\mathbf{J}_{p} .
$$

Taking the divergence gives

By Faraday's law of induction,

$$
\epsilon \nabla \cdot \frac{\partial \mathbf{E}}{\partial t}=-\nabla \cdot\left(\sigma_{a} \mathbf{E}\right)-\nabla \cdot \mathbf{J}_{p}
$$

$$
\operatorname{curl}(\mathbf{E})=-\frac{\partial \mathbf{B}}{\partial t}
$$

where $\mathbf{B}$ is the magnetic flux density. Assuming the time derivative of $\mathbf{B}$ can be neglected, we have curl $(\mathbf{E})=\mathbf{0}$, which implies that $\mathbf{E}$ can be represented as the gradient of a potential. It is common to write $\mathbf{E}=-\nabla \phi$. With this substitution, (2.1) reduces to (1.1) where $\sigma=\sigma_{a} / \epsilon$ and $\mathbf{J}=\mathbf{J}_{p} / \epsilon$.

\section{LIMIT IN THE DISCRETE EQUATIONS}

In this section, we will prove Theorem 1.2. The solution to (1.13) is given by

$$
\boldsymbol{\Phi}(t, \tau)=\mathrm{e}^{-t \mathbf{A}^{-1}\left(\mathbf{B}+\tau \mathbf{U U}^{\top}\right)} \boldsymbol{\Phi}_{0}-\left(\int_{0}^{t} \mathrm{e}^{(s-t) \mathbf{A}^{-1}\left(\mathbf{B}+\tau \mathbf{U} \mathbf{U}^{\top}\right)} \mathrm{d} s\right) \mathbf{A}^{-1} \mathbf{i} .
$$

If the matrices $\mathbf{A}^{-1} \mathbf{B}$ and $\mathbf{A}^{-1} \mathbf{U} \mathbf{U}^{\top}$ commute, then we could write

$$
\mathrm{e}^{-t \mathbf{A}^{-1}\left(\mathbf{B}+\tau \mathbf{U} \mathbf{U}^{\top}\right)}=\mathrm{e}^{-t \mathbf{A}^{-1} \mathbf{B}} \times \mathrm{e}^{-t \tau \mathbf{A}^{-1} \mathbf{U U}^{\top}},
$$

and the analysis of the limit, as $\tau$ tends to $\infty$, could be computed using the simple proof given in [4]. However, in general, when $\mathbf{A}$ might be the matrix associated with a discretization of the Laplacian, the matrices $\mathbf{A}^{-1} \mathbf{B}$ and $\mathbf{A}^{-1} \mathbf{U U}^{\top}$ are not expected to commute. The analysis which follows provides a strategy for obtaining the same limit given in [4] without assuming commutativity.

Suppose that $\mathbf{X}$ and $\mathbf{Y}$ are $n$ by $n$ matrices with $\mathbf{X}$ symmetric and positive definite. By a Taylor expansion for the exponential, we have

$$
\begin{aligned}
\mathrm{e}^{\mathbf{X Y}} & =\sum_{i=0}^{\infty} \frac{(\mathbf{X Y})^{k}}{k !}=\mathbf{X}^{1 / 2}\left(\sum_{i=0}^{\infty} \frac{\left(\mathbf{X}^{1 / 2} \mathbf{Y} \mathbf{X}^{1 / 2}\right)^{k}}{k !}\right) \mathbf{X}^{-1 / 2} \\
& =\mathbf{X}^{1 / 2}\left(\mathrm{e}^{\mathbf{X}^{1 / 2} \mathbf{Y} \mathbf{X}^{1 / 2}}\right) \mathbf{X}^{-1 / 2}
\end{aligned}
$$

Hence, we have

$$
\begin{aligned}
\mathrm{e}^{-t \mathbf{A}^{-1}\left(\mathbf{B}+\tau \mathbf{U U}^{\top}\right)} & =\mathbf{A}^{-1 / 2}\left(\mathrm{e}^{-t\left(\mathbf{A}^{-1 / 2} \mathbf{B} \mathbf{A}^{-1 / 2}+\tau \mathbf{A}^{-1 / 2} \mathbf{U U}^{\top} \mathbf{A}^{-1 / 2}\right)}\right) \mathbf{A}^{1 / 2} \\
& =\mathbf{A}^{-1 / 2}\left(\mathrm{e}^{-t\left(\mathbf{C}+\tau \mathbf{V} \mathbf{V}^{\top}\right)}\right) \mathbf{A}^{1 / 2}
\end{aligned}
$$


where

$$
\mathbf{C}=\mathbf{A}^{-1 / 2} \mathbf{B A}^{-1 / 2} \text { and } \mathbf{V}=\mathbf{A}^{-1 / 2} \mathbf{U}
$$

Let $\mathbf{W} \boldsymbol{\Lambda} \mathbf{W}^{\top}$ be a diagonalization of $\mathbf{V} \mathbf{V}^{\top}$ where $\mathbf{W}$ is orthogonal and the diagonal elements of $\boldsymbol{\Lambda}$ are arranged in decreasing order:

$$
\lambda_{1} \geq \lambda_{2} \geq \ldots \geq \lambda_{m}>0 \quad \text { and } \quad \lambda_{i}=0 \text { for } i>m .
$$

There are precisely $m$ positive eigenvalues since $\mathbf{U}$ and $\mathbf{V}$ have rank $m$. Replacing $\mathbf{V} \mathbf{V}^{\top}$ by its diagonalization gives

$$
\mathrm{e}^{-t\left(\mathbf{C}+\tau \mathbf{V} \mathbf{V}^{\top}\right)}=\mathrm{e}^{-t\left(\mathbf{C}+\tau \mathbf{W} \boldsymbol{\Lambda} \mathbf{W}^{\top}\right)}=\mathbf{W}\left(\mathrm{e}^{-t\left(\mathbf{W}^{\top} \mathbf{C W}+\tau \boldsymbol{\Lambda}\right)}\right) \mathbf{W}^{\top} .
$$

If $\mathbf{Q}(\tau) \mathbf{D}(\tau) \mathbf{Q}(\tau)^{\top}$ is a diagonalization of $\mathbf{W}^{\top} \mathbf{C W}+\tau \boldsymbol{\Lambda}$, with eigenvalues arranged in decreasing order, then

$$
\begin{aligned}
\mathrm{e}^{-t\left(\mathbf{W}^{\top} \mathbf{C W}+\tau \boldsymbol{\Lambda}\right)} & =\mathrm{e}^{-t \mathbf{Q}(\tau) \mathbf{D}(\tau) \mathbf{Q}(\tau)^{\top}} \\
& =\mathbf{Q}(\tau) \mathrm{e}^{-t \mathbf{D}(\tau)} \mathbf{Q}(\tau)^{\top} \\
& =\mathbf{I}+\mathbf{Q}(\tau)\left(\mathrm{e}^{-t \mathbf{D}(\tau)}-\mathbf{I}\right) \mathbf{Q}(\tau)^{\top} \\
& =\mathbf{I}+\mathbf{Q}(\tau)\left(\left[\mathrm{e}^{-t \tau \boldsymbol{\Lambda}}\right]\left[\mathrm{e}^{-t(\mathbf{D}(\tau)-\tau \boldsymbol{\Lambda})}\right]-\mathbf{I}\right) \mathbf{Q}(\tau)^{\top}
\end{aligned}
$$

where $\mathbf{I}$ denotes the $n$ by $n$ identity matrix.

By a result [11], p. 191, concerning the perturbation in eigenvalues for symmetric matrices, we have

$$
\|\mathbf{D}(\tau)-\tau \mathbf{\Lambda}\|=\max _{1 \leq i \leq n}\left|d_{i i}(\tau)-\tau \lambda_{i}\right| \leq\|\mathbf{C}\|
$$

That is, the $i$ th largest eigenvalue $d_{i i}(\tau)$ of $\mathbf{W}^{\top} \mathbf{C W}+\tau \boldsymbol{\Lambda}$ differs from the $i$ th largest eigenvalue $\tau \lambda_{i}$ of $\tau \boldsymbol{\Lambda}$ by at most $\left\|\mathbf{W}^{\top} \mathbf{C W}\right\|=\|\mathbf{C}\|$.

For any square matrix $\mathbf{M}$, the triangle inequality yields

$$
\left\|\mathbf{I}-\mathrm{e}^{\mathbf{M}}\right\|=\left\|\sum_{i=1}^{\infty} \frac{\mathbf{M}^{k}}{k !}\right\| \leq \sum_{i=1}^{\infty} \frac{\|\mathbf{M}\|^{k}}{k !}=\mathrm{e}^{\|\mathbf{M}\|}-1 .
$$

Combine this with (3.6) to obtain

$$
\left\|\mathbf{I}-\mathrm{e}^{-t(\mathbf{D}(\tau)-\tau \boldsymbol{\Lambda})}\right\| \leq \mathrm{e}^{t\|\mathbf{C}\|}-1
$$

Hence, uniformly in $\tau$, we have

$$
\lim _{t \rightarrow 0^{+}} \mathrm{e}^{-t(\mathbf{D}(\tau)-\tau \boldsymbol{\Lambda})}=\mathbf{I}
$$

For any $t>0$, it follows from (3.3) that

$$
\lim _{\tau \rightarrow \infty} \mathrm{e}^{-t \tau \boldsymbol{\Lambda}}=\left[\begin{array}{ll}
\mathbf{0}_{m \times m} & \mathbf{0}_{m \times(n-m)} \\
\mathbf{0}_{(n-m) \times m} & \mathbf{I}_{(n-m) \times(n-m)}
\end{array}\right]:=\mathbf{I}_{0},
$$

where the subscripts on the matrices denote their dimensions, and $\mathbf{0}$ is a matrix whose entries are all zero. Combine (3.5), (3.7), and (3.8) to obtain

$$
\begin{aligned}
\mathrm{e}^{-t\left(\mathbf{W}^{\top} \mathbf{C W}+\tau \boldsymbol{\Lambda}\right)} & =\mathbf{I}+\mathbf{Q}(\tau)\left(\mathbf{I}_{0}-\mathbf{I}+\mathbf{R}(t, \tau)\right) \mathbf{Q}(\tau)^{\top} \\
& =\mathbf{Q}(\tau)\left(\mathbf{I}_{0}+\mathbf{R}(t, \tau)\right) \mathbf{Q}(\tau)^{\top},
\end{aligned}
$$

where the remainder term $\mathbf{R}$ has the property that

$$
\lim _{t \rightarrow 0^{+}} \lim _{\tau \rightarrow \infty} \mathbf{R}(t, \tau)=\mathbf{0} .
$$


Suppose $\mathbf{Q}(\tau)$ is block partitioned in accordance with (3.8):

$$
\mathbf{Q}(\tau)=\left[\begin{array}{ll}
\mathbf{Q}_{11}(\tau) & \mathbf{Q}_{12}(\tau) \\
\mathbf{Q}_{21}(\tau) & \mathbf{Q}_{22}(\tau)
\end{array}\right]
$$

The eigenvector matrix $\mathbf{Q}(\tau)$ for $\mathbf{W}^{\top} \mathbf{C W}+\tau \boldsymbol{\Lambda}$, is the same as the eigenvector matrix for $\mathbf{W}^{\top} \mathbf{C W} / \tau+\boldsymbol{\Lambda}$. Since the eigenvector matrix for $\boldsymbol{\Lambda}$ is $\mathbf{I}$, it follows that as $\tau$ tends to infinity, $\mathbf{Q}_{21}(\tau)$ and $\mathbf{Q}_{12}(\tau)$ approach zero. Since $\mathbf{Q}(\tau)$ is orthogonal, we have

$$
\begin{aligned}
\lim _{\tau \rightarrow \infty} \mathbf{Q}_{22}(\tau) \mathbf{Q}_{22}(\tau)^{\top} & =\mathbf{I}_{(n-m) \times(n-m)}, \\
\lim _{\tau \rightarrow \infty} \mathbf{Q}_{21}(\tau) & =\mathbf{0}_{(n-m) \times m}, \\
\lim _{\tau \rightarrow \infty} \mathbf{Q}_{12}(\tau) & =\mathbf{0}_{m \times(n-m)} .
\end{aligned}
$$

Hence, $\mathbf{Q}(\tau) \mathbf{I}_{0} \mathbf{Q}(\tau)^{\top}$ approaches $\mathbf{I}_{0}$ as $\tau$ tends to infinity, and by (3.4) and (3.9), we conclude that

$$
\lim _{t \rightarrow 0^{+}} \lim _{\tau \rightarrow \infty} \mathrm{e}^{-t\left(\mathbf{C}+\tau \mathbf{V} \mathbf{V}^{\top}\right)}=\mathbf{W I}_{0} \mathbf{W}^{\top}=\mathbf{W}\left(\mathbf{I}+\left(\mathbf{I}_{0}-\mathbf{I}\right)\right) \mathbf{W}^{\top}=\mathbf{I}-\mathbf{W}_{1} \mathbf{W}_{1}^{\top},
$$

where $\mathbf{W}_{1}$ is the submatrix of $\mathbf{W}$ corresponding to the first $m$ columns. Combining this with (3.2) gives

$$
\begin{aligned}
\lim _{t \rightarrow 0^{+}} \lim _{\tau \rightarrow \infty} \mathrm{e}^{-t \mathbf{A}^{-1}\left(\mathbf{B}+\tau \mathbf{U U}^{\top}\right)} & =\mathbf{A}^{-1 / 2}\left(\mathbf{I}-\mathbf{W}_{1} \mathbf{W}_{1}^{\top}\right) \mathbf{A}^{1 / 2} \\
& =\mathbf{I}-\mathbf{A}^{-1 / 2} \mathbf{W}_{1} \mathbf{W}_{1}^{\top} \mathbf{A}^{1 / 2}
\end{aligned}
$$

Recall that $\mathbf{W}_{1}$ is a matrix whose columns are orthonormal eigenvectors associated with the $m$ nonzero eigenvalues of $\mathbf{V V}^{\top}$. We now show that

$$
\mathbf{W}_{1}=\mathbf{P} \quad \text { where } \quad \mathbf{P}=\mathbf{V X} \boldsymbol{\Sigma}^{-1 / 2}
$$

and $\mathbf{X} \boldsymbol{\Sigma} \mathbf{X}^{\top}$ is a diagonalization of $\mathbf{V}^{\top} \mathbf{V}$ with the eigenvalues arranged in decreasing order. First, observe that the columns of $\mathbf{P}$ are orthonormal since

$$
\mathbf{P}^{\top} \mathbf{P}=\boldsymbol{\Sigma}^{-1 / 2} \mathbf{X}^{\top} \mathbf{V}^{\top} \mathbf{V} \mathbf{X} \boldsymbol{\Sigma}^{-1 / 2}=\boldsymbol{\Sigma}^{-1 / 2} \mathbf{X}^{\top} \mathbf{X} \boldsymbol{\Sigma} \mathbf{X}^{\top} \mathbf{X} \boldsymbol{\Sigma}^{-1 / 2}=\mathbf{I}_{m \times m} .
$$

The columns of $\mathbf{P}$ are eigenvectors of $\mathbf{V} \mathbf{V}^{\top}$ since

$$
\begin{aligned}
\mathbf{V} \mathbf{V}^{\top} \mathbf{P} & =\mathbf{V} \mathbf{V}^{\top} \mathbf{V X} \boldsymbol{\Sigma}^{-1 / 2}=\mathbf{V} \mathbf{X} \boldsymbol{\Sigma} \mathbf{X}^{\top} \mathbf{X} \boldsymbol{\Sigma}^{-1 / 2} \\
& =\mathbf{V X} \boldsymbol{\Sigma}^{1 / 2}=\mathbf{V X} \boldsymbol{\Sigma}^{-1 / 2} \boldsymbol{\Sigma}=\mathbf{P} \boldsymbol{\Sigma} .
\end{aligned}
$$

We utilize (3.11) and the substitution $\mathbf{V}=\mathbf{A}^{-1 / 2} \mathbf{U}$ to obtain

$$
\begin{aligned}
\mathbf{W}_{1} \mathbf{W}_{1}^{\top} & =\mathbf{V X} \boldsymbol{\Sigma}^{-1 / 2} \boldsymbol{\Sigma}^{-1 / 2} \mathbf{X}^{\top} \mathbf{V}^{\top}=\mathbf{V X} \boldsymbol{\Sigma}^{-1} \mathbf{X}^{\top} \mathbf{V}^{\top} \\
& =\mathbf{V}\left(\mathbf{V}^{\top} \mathbf{V}\right)^{-1} \mathbf{V}^{\top}=\mathbf{A}^{-1 / 2} \mathbf{U}\left(\mathbf{U}^{\top} \mathbf{A}^{-1} \mathbf{U}\right)^{-1} \mathbf{U}^{\top} \mathbf{A}^{-1 / 2}
\end{aligned}
$$

Referring to (3.10), we have

$$
\lim _{t \rightarrow 0+\tau \rightarrow \infty} \lim _{\tau \rightarrow \mathrm{A}^{-1}\left(\mathbf{B}+\tau \mathbf{U} \mathbf{U}^{\top}\right)}=\mathbf{I}-\mathbf{A}^{-1} \mathbf{U}\left(\mathbf{U}^{\top} \mathbf{A}^{-1} \mathbf{U}\right)^{-1} \mathbf{U}^{\top} .
$$

By the uniform boundedness of the exponential in (3.9), the integral term in (3.1) tends to zero as $t$ tends to 0 . Relations (3.1) and (3.12) complete the proof of Theorem 1.2. 


\section{Reformulation of the CONTINUOUs EQUATion}

Let $\left(\phi_{i}, \lambda_{i}\right), i \in \mathbb{N}$, denote a complete orthonormal set of eigenfunctions for the generalized eigenproblem (1.15), as given by Theorem 1.3. We decompose $\mathbb{N}$ into the disjoint union of four sets corresponding to the four classes of eigenfunctions described in the introduction:

$$
\begin{aligned}
\mathcal{S}_{\Pi} & =\left\{i \in \mathbb{N}: \phi_{i}=\Pi /\|\nabla \Pi\|_{L^{2}(\Omega)}\right\}, \\
\mathcal{S}_{0} & =\left\{i \in \mathbb{N}: \lambda_{i}=0, \quad\left\langle\nabla \phi_{i}, \nabla \Pi\right\rangle_{\Omega}=0\right\}, \\
\mathcal{S}_{1} & =\left\{i \in \mathbb{N}: \lambda_{i}=1\right\}, \\
\mathcal{S}_{+} & =\left\{i \in \mathbb{N}: 0<\lambda_{i}<1\right\} .
\end{aligned}
$$

The set $\mathcal{S}_{\Pi}$ contains precisely one element corresponding to the eigenfunction $\Pi$ given by (1.9). The set $\mathcal{S}_{0}$ corresponds to eigenfunctions supported on $\mathcal{L}^{c}$, while $\mathcal{S}_{1}$ corresponds to eigenfunctions supported on $\mathcal{L}$. The set $\mathcal{S}_{+}$corresponds to functions in $H_{0}^{1}(\Omega)$ which are harmonic in both $\mathcal{L}$ and $\mathcal{L}^{c}$. The eigenvalues associated with indices in $\mathcal{S}_{+}$are uniformly bounded away from 0 by [2], Proposition 6.1.

The weak form of (1.5) is to find $\phi$ such that

$$
\frac{\partial}{\partial t}\langle\nabla \phi, \nabla v\rangle_{\Omega}=-\langle\sigma \nabla \phi, \nabla v\rangle_{\Omega}-\tau\langle\nabla \phi, \nabla v\rangle_{\mathcal{L}}+\langle\mathbf{J}, \nabla v\rangle_{\Omega}
$$

for all $v \in H_{0}^{1}(\Omega)$. We substitute the eigenexpansion

$$
\phi(\mathbf{x}, t)=\sum_{i \in \mathbb{N}} \alpha_{i}(t) \phi_{i}(\mathbf{x})
$$

into (4.1). Taking $v=\phi_{j}, j=1,2, \ldots$, and utilizing the orthonormality of the eigenfunctions yields the linear system

$$
\dot{\boldsymbol{\alpha}}=-\mathbf{A} \boldsymbol{\alpha}-\tau \mathbf{D} \boldsymbol{\alpha}+\mathbf{f}, \quad \boldsymbol{\alpha}(0)=\boldsymbol{\alpha}_{0},
$$

where

$$
\begin{aligned}
\left(\boldsymbol{\alpha}_{0}\right)_{i} & =\left\langle\nabla \phi_{0}, \nabla \phi_{i}\right\rangle_{\Omega}, \quad i \in \mathbb{N}, \\
a_{i j} & =\left\langle\sigma \nabla \phi_{i}, \nabla \phi_{j}\right\rangle_{\Omega}, \\
d_{i j} & =\left\langle\nabla \phi_{i}, \nabla \phi_{j}\right\rangle_{\mathcal{L}}, \\
f_{i} & =\left\langle\mathbf{J}, \nabla \phi_{i}\right\rangle_{\Omega} .
\end{aligned}
$$

Since the $\phi_{i}$ are orthonormal eigenfunctions for (1.15), we have

$$
d_{i j}=\left\langle\nabla \phi_{i}, \nabla \phi_{j}\right\rangle_{\mathcal{L}}=\lambda_{i}\left\langle\phi_{i}, \phi_{j}\right\rangle_{\Omega}= \begin{cases}\lambda_{i} & \text { if } i=j \\ 0 & \text { otherwise }\end{cases}
$$

Hence, $\mathbf{D}$ is a diagonal matrix with the eigenvalues $\lambda_{i}, i \in \mathbb{N}$, on the diagonal. Since the eigenvalues are nonnegative, $\mathbf{D}$ is positive semidefinite. We now consider $\mathbf{A}$ and $\mathbf{f}$ :

Lemma 4.1. The matrix $\mathbf{A}$ is positive semidefinite. The 2-norms of $\mathbf{A}$ and $\mathbf{f}$, defined in (4.5) and (4.6) respectively, are both finite, and we have

$$
\|\mathbf{A}\| \leq \operatorname{ess} \sup _{\mathbf{x} \in \Omega}|\sigma(\mathbf{x})|:=\|\sigma\|_{L^{\infty}(\Omega)} \quad \text { and } \quad\|\mathbf{f}\| \leq\|\mathbf{J}\|_{L^{2}(\Omega)} .
$$

The 2-norm of $\mathbf{D}$ satisfies $\|\mathbf{D}\| \leq 1$. 
Proof. Since the eigenvalues of the generalized eigenproblem (1.15) satisfy $0 \leq \lambda_{i} \leq 1$, and since $\mathbf{D}$ is a diagonal matrix with the eigenvalues on the diagonal, the inequality $\|\mathbf{D}\| \leq 1$ follows immediately. By the definition of $\mathbf{A}$, we have

$$
\mathbf{x}^{\top} \mathbf{A} \mathbf{x}=\left\langle\sigma \sum_{i=1}^{\infty} x_{i} \nabla \phi_{i}, \sum_{i=1}^{\infty} x_{i} \nabla \phi_{i}\right\rangle_{\Omega} \geq 0
$$

for all $\mathbf{x} \in \ell^{2}$ since $\sigma \geq 0$. Since $\mathbf{A}$ is positive semidefinite, the Cauchy-Schwarz inequality yields

$$
\left|\mathbf{y}^{\top} \mathbf{A x}\right| \leq \sqrt{\mathbf{y}^{\top} \mathbf{A y}} \sqrt{\mathbf{x}^{\top} \mathbf{A x}}
$$

for all $\mathbf{x}$ and $\mathbf{y} \in \ell^{2}$. If $\|\mathbf{x}\|=1$, then by (4.8) we have

$$
\mathbf{x}^{\top} \mathbf{A} \mathbf{x} \leq\|\sigma\|_{L^{\infty}}\left\langle\sum_{i=1}^{\infty} x_{i} \nabla \phi_{i}, \sum_{i=1}^{\infty} x_{i} \nabla \phi_{i}\right\rangle_{\Omega}=\|\sigma\|_{L^{\infty}} .
$$

Hence, (4.9) yields the first inequality in (4.7).

Let $u \in H_{0}^{1}(\Omega)$ be the weak solution to $\Delta u=-\nabla \cdot \mathbf{J}$ :

$$
\langle\nabla u, \nabla v\rangle=\langle\mathbf{J}, \nabla v\rangle \quad \text { for all } v \in H_{0}^{1}(\Omega) .
$$

We have

Dividing by $\|\nabla u\|$ gives

$$
\|\nabla u\|^{2}=\langle\nabla u, \nabla u\rangle=\langle\mathbf{J}, \nabla u\rangle \leq\|\mathbf{J}\|\|\nabla u\| .
$$

$$
\|\nabla u\| \leq\|\mathbf{J}\|
$$

For $\mathbf{f}$ defined in (4.6),

$$
\|\mathbf{f}\|^{2}=\sum_{i=1}^{\infty}\left\langle\mathbf{J}, \nabla \phi_{i}\right\rangle_{\Omega}^{2}=\sum_{i=1}^{\infty}\left\langle\nabla u, \nabla \phi_{i}\right\rangle_{\Omega}^{2}=\langle\nabla u, \nabla u\rangle_{\Omega} \leq\|\mathbf{J}\|^{2} .
$$

The last equality is due to the fact that the $\phi_{i}$ are a complete orthonormal basis relative to the $H_{0}^{1}(\Omega)$ inner product, while the last inequality is (4.10).

Theorem 4.2. Equation (4.3) has a unique solution $\boldsymbol{\alpha}(\cdot):[0, \infty) \rightarrow \ell^{2}$ with $\boldsymbol{\alpha}(\cdot)$ and $\dot{\boldsymbol{\alpha}}(\cdot)$ continuous on $[0, \infty)$.

Proof. By the boundedness of $\|\mathbf{A}\|$ and $\|\mathbf{D}\|$ (Lem. 4.1), it follows from [3], Corollary 2.2.3, that $-(\mathbf{A}+\tau \mathbf{D})$ is the infinitesimal generator for a strongly continuous semigroup $\mathrm{e}^{-(\mathbf{A}+\tau \mathbf{D})}$ on $\ell^{2}$. Hence, by [1], Theorem 7.10, equation (4.3) has a unique solution which is continuous and which has a continuous time derivative on $[0, \infty)$.

\section{Potential Change for the Continuous equation}

We now prove Theorem 1.1. Multiply (4.3) by $\boldsymbol{\alpha}^{\top}$ and utilize the fact that $\mathbf{D}$ and $\mathbf{A}$ are positive semidefinite to obtain

Hence, we have

$$
\boldsymbol{\alpha}^{\top} \dot{\boldsymbol{\alpha}}=-\boldsymbol{\alpha}^{\top} \mathbf{A} \boldsymbol{\alpha}-\tau \boldsymbol{\alpha}^{\top} \mathbf{D} \boldsymbol{\alpha}+\boldsymbol{\alpha}^{\top} \mathbf{f} \leq \boldsymbol{\alpha}^{\top} \mathbf{f} .
$$

Multiplying by $\mathrm{e}^{-t}$ and rearranging yields

$$
\frac{1}{2} \frac{\mathrm{d}}{\mathrm{d} t}\|\boldsymbol{\alpha}\|^{2}=\boldsymbol{\alpha}^{\top} \dot{\boldsymbol{\alpha}} \leq\|\boldsymbol{\alpha}\|\|\mathbf{f}\| \leq \frac{1}{2}\left(\|\boldsymbol{\alpha}\|^{2}+\|\mathbf{f}\|^{2}\right) .
$$

$$
\frac{\mathrm{d}}{\mathrm{d} t}\left(\mathrm{e}^{-t}\|\boldsymbol{\alpha}\|^{2}\right) \leq \mathrm{e}^{-t}\|\mathbf{f}\|^{2} .
$$


Integration over the interval $[0, t]$ gives

$$
\|\boldsymbol{\alpha}(t)\|^{2} \leq \mathrm{e}^{t}\|\boldsymbol{\alpha}(0)\|^{2}+\left(\mathrm{e}^{t}-1\right)\|\mathbf{f}\|^{2} .
$$

This shows that $\|\boldsymbol{\alpha}(\cdot)\|$ is uniformly bounded over any finite interval.

For the remaining analysis, it is convenient to group the eigenvectors into two sets, corresponding to eigenvectors whose associated eigenvalues are positive (the index sets $\mathcal{S}_{1}$ and $\mathcal{S}_{+}$) and eigenvectors whose associated eigenvalues are zero (the index sets $\mathcal{S}_{\Pi}$ and $\mathcal{S}_{0}$ ). This grouping of the eigenvectors corresponds to a permutation of $\mathbf{D}$ which has the following structure:

$$
\mathbf{D}=\left[\begin{array}{ll}
\Lambda & 0 \\
0 & 0
\end{array}\right]
$$

where $\boldsymbol{\Lambda}$ is a diagonal matrix with strictly positive diagonal and the $\mathbf{0}$ 's represent infinite blocks whose elements are all zero. The components of $\boldsymbol{\alpha}$ are partitioned in a compatible way into $[\mathbf{p} ; \mathbf{z}]$ where $\mathbf{p}$ corresponds to the positive eigenvalues $\lambda_{i}$ and $\mathbf{z}$ corresponds to the zero eigenvalues.

Multiply $(4.3)$ by $\left[\mathbf{p}^{\top} ; \mathbf{0}\right]$ to obtain

$$
\begin{aligned}
\mathbf{p}^{\top} \dot{\mathbf{p}} & =[\mathbf{p} ; \mathbf{0}]^{\top} \dot{\boldsymbol{\alpha}} \\
& =-[\mathbf{p} ; \mathbf{0}]^{\top} \mathbf{A} \boldsymbol{\alpha}-\tau[\mathbf{p} ; \mathbf{0}]^{\top}\left[\begin{array}{c}
\boldsymbol{\Lambda} \mathbf{p} \\
\mathbf{0}
\end{array}\right]+[\mathbf{p} ; \mathbf{0}]^{\top} \mathbf{f} .
\end{aligned}
$$

Taking norms on the right side gives

$$
\begin{aligned}
\frac{1}{2} \frac{\mathrm{d}}{\mathrm{d} t}\|\mathbf{p}\|^{2} & \leq\|\boldsymbol{\alpha}\|^{2}\|\mathbf{A}\|-\tau \mathbf{p}^{\top} \boldsymbol{\Lambda} \mathbf{p}+\|\mathbf{p}\|\|\mathbf{f}\| \\
& \leq\|\boldsymbol{\alpha}\|^{2}\|\mathbf{A}\|-\tau \lambda_{0}\|\mathbf{p}\|^{2}+\frac{1}{2}\left(\|\mathbf{p}\|^{2}+\|\mathbf{f}\|^{2}\right)
\end{aligned}
$$

where $\lambda_{0}$ denotes the smallest positive eigenvalue; a positive lower bound for $\lambda_{0}$ is obtained in [2], Proposition 6.1. Suppose $\tau$ is large enough that $\tau \lambda_{0} \geq 1$. Choose $t>0$ and let $c$ be the constant defined by

$$
c=\|\mathbf{f}\|^{2}+2\|\mathbf{A}\| \max _{s \in[0, t]}\|\boldsymbol{\alpha}(s)\|^{2}
$$

which is finite due to (5.1). By (5.2), we have

$$
\frac{\mathrm{d}}{\mathrm{d} t}\|\mathbf{p}\|^{2} \leq c-\tau \lambda_{0}\|\mathbf{p}\|^{2}
$$

on the interval $[0, t]$ assuming $\tau \lambda_{0} \geq 1$. Multiplying both sides by $\mathrm{e}^{\tau \lambda_{0} t}$ yields

$$
\frac{\mathrm{d}}{\mathrm{d} t}\left(\mathrm{e}^{\tau \lambda_{0} t}\|\mathbf{p}\|^{2}\right) \leq \mathrm{e}^{\tau \lambda_{0} t} c
$$

Integration over the interval $[0, t]$ gives

$$
\begin{aligned}
\|\mathbf{p}(t)\|^{2} & \leq \mathrm{e}^{-\tau \lambda_{0} t}\|\mathbf{p}(0)\|^{2}+\frac{1}{\tau \lambda_{0}}\left(1-\mathrm{e}^{-\tau \lambda_{0} t}\right) c \\
& \leq \mathrm{e}^{-\tau \lambda_{0} t}\|\mathbf{p}(0)\|^{2}+\frac{c}{\tau \lambda_{0}}
\end{aligned}
$$

Since the right side approaches 0 as $\tau$ tends to $\infty$, we conclude that for any $t>0$,

$$
\lim _{\tau \rightarrow \infty} \mathbf{p}_{\tau}(t)=\mathbf{0}
$$


Here we have inserted a $\tau$ subscript on $\mathbf{p}$ to remind us that the $\mathbf{p}$-component of the solution $\boldsymbol{\alpha}$ to (4.3) depends on $\tau$.

Now consider the bottom half of equation (4.3):

$$
\dot{\mathbf{z}}_{\tau}=\mathbf{A}_{2} \boldsymbol{\alpha}_{\tau}-\mathbf{f}_{2},
$$

where $\mathbf{A}_{2}$ denotes the bottom half of $\mathbf{A}$ and $\mathbf{f}_{2}$ is the bottom half of $\mathbf{f}$. Since the bottom half of $\mathbf{D}$ is zero, the $\mathbf{D}$ term of (4.3) is not present in (5.4). Integrating (5.4) over the interval $(0, t)$ yields

$$
\mathbf{z}_{\tau}(t)=\mathbf{z}(0)+\mathbf{r}_{\tau}(t), \quad \mathbf{r}_{\tau}(t):=\int_{0}^{t}\left(\mathbf{A}_{2} \boldsymbol{\alpha}_{\tau}(s)-\mathbf{f}_{2}\right) \mathrm{d} s,
$$

where $\mathbf{z}(0)$ is the vector of coefficients in the eigenfunction expansion of $\phi_{0}$ corresponding to the eigenvalues $\lambda_{i}=0$. For any given $T>0$, it follows from (5.1) that there exists a constant $c$ such that $\left\|\boldsymbol{\alpha}_{\tau}(t)\right\| \leq c$ for all $t \in[0, T]$. Hence, for any $t \in[0, T]$, we have

$$
\left\|\mathbf{r}_{\tau}(t)\right\| \leq t\left(\left\|\mathbf{A}_{2}\right\| c+\left\|\mathbf{f}_{2}\right\|\right),
$$

independent of $\tau$. Consequently, $\mathbf{r}_{\tau}(t)$ tends to $\mathbf{0}$ as $t$ tends to 0 , independent of $\tau$. By (5.5) we conclude that $\mathbf{z}_{\tau}(t)$ converges to $\mathbf{z}(0)$ in $\ell_{2}$ as $t$ tends to 0 , independent of $\tau$.

In summary, the vector $\boldsymbol{\alpha}_{\tau}(t)=\left[\mathbf{p}_{\tau}(t) ; \mathbf{z}_{\tau}(t)\right]$ has the property that $\mathbf{p}_{\tau}(t)$ tends to $\mathbf{0}$ as $\tau$ tends to infinity for any fixed $t$ (see (5.3)), while $\mathbf{z}_{\tau}(t)$ tends to $\mathbf{z}(0)$ as $t$ tends to zero, independent of $\tau$. More succinctly,

$$
\lim _{t \rightarrow 0^{+}} \lim _{\tau \rightarrow \infty} \boldsymbol{\alpha}_{\tau}(t)=[\mathbf{0} ; \mathbf{z}(0)]
$$

We now exploit these observations to analyze the expansion for $\phi$ in (4.2):

$$
\phi_{\tau}(\mathbf{x}, t)=\sum_{i \in \mathcal{S}_{+} \cup \mathcal{S}_{1}} \alpha_{\tau, i}(t) \phi_{i}(\mathbf{x})+\sum_{i \in \mathcal{S}_{0} \cup \mathcal{S}_{\Pi}} \alpha_{\tau, i}(t) \phi_{i}(\mathbf{x}) .
$$

The coefficients $\alpha_{\tau, i}(t)$ for $i \in \mathcal{S}_{+} \cup \mathcal{S}_{1}$ are components of the vector $\mathbf{p}_{\tau}(t)$, which tend to 0 as $\tau$ tends to infinity by (5.3). The coefficients $\alpha_{\tau, i}(t)$ for $i \in \mathcal{S}_{0} \cup \mathcal{S}_{\Pi}$ are components of the vector $\mathbf{z}_{\tau}(t)$, which approaches $\mathbf{z}(0)$, as $t$ tends to 0 , independent of $\tau$. Since the components of $\mathbf{z}(0)$ are the coefficients in the eigenfunction expansion of $\phi_{0}$ corresponding to the eigenvalues $\lambda_{i}=0$, we have

$$
\phi^{+}(x)=\lim _{t \rightarrow 0^{+}} \lim _{\tau \rightarrow \infty} \phi_{\tau}(\mathbf{x}, t)=\sum_{i \in \mathcal{S}_{\Pi} \cup \mathcal{S}_{0}} \alpha_{i}(0) \phi_{i}(x),
$$

where

$$
\alpha_{i}(0)=\left\langle\nabla \phi_{0}, \nabla \phi_{i}\right\rangle_{\Omega}
$$

For $\mathbf{x} \in \mathcal{L}$ and $i \in \mathcal{S}_{0}, \phi_{i}(\mathbf{x})=0$ since $\phi_{i}, i \in \mathcal{S}_{0}$, is supported on $\mathcal{L}^{c}$. Hence, for $\mathbf{x} \in \mathcal{L}$, we have

$$
\phi^{+}(x)=\sum_{i \in \mathcal{S}_{\Pi}} \alpha_{i}(0) \phi_{i}(x)
$$

Since $\phi_{i}$ for $i \in \mathcal{S}_{\Pi}$ is the normalized $\Pi, \alpha_{i}(0) \phi_{i}(x)$ is simply the projection of $\phi_{0}$ along $\Pi$ :

$$
\sum_{i \in \mathcal{S}_{\Pi}} \alpha_{i}(0) \phi_{i}(x)=\left(\frac{\left\langle\nabla \phi_{0}, \nabla \Pi\right\rangle_{\Omega}}{\langle\nabla \Pi, \nabla \Pi\rangle_{\Omega}}\right) \Pi(\mathbf{x})=\phi_{L} \Pi(\mathbf{x}) .
$$

Since $\Pi(\mathbf{x})=1$ for $\mathbf{x} \in \mathcal{L}$, the top half of (1.7) has been established. 
Now suppose that $\mathbf{x} \in \mathcal{L}^{c}$. By the completeness of the $\phi_{i}$, we have

$$
\phi_{0}(\mathbf{x})=\sum_{i \in \mathbb{N}} \alpha_{i}(0) \phi_{i}(\mathbf{x})
$$

Consequently, for $\mathbf{x} \in \mathcal{L}^{c},(5.6)$ can be rewritten

$$
\phi^{+}(\mathbf{x})=\phi_{0}(\mathbf{x})-\sum_{i \in \mathcal{S}_{1} \cup \mathcal{S}_{+}} \alpha_{i}(0) \phi_{i}(\mathbf{x})=\phi_{0}(\mathbf{x})-\sum_{i \in \mathcal{S}_{+}} \alpha_{i}(0) \phi_{i}(\mathbf{x})
$$

since $\phi_{i}$ for $i \in \mathcal{S}_{1}$ vanishes on $\mathcal{L}^{c}$. Let $\xi$ denote the final term in (5.9):

$$
\xi(x)=-\sum_{i \in \mathcal{S}_{+}} \alpha_{i}(0) \phi_{i}(\mathbf{x}) .
$$

For $i \in \mathcal{S}_{+}$, we have $\Delta \phi_{i}=0$ on $\mathcal{L}^{c}$ since the eigenfunctions associated with indices in $\mathcal{S}_{+}$are harmonic in either $\mathcal{L}$ or $\mathcal{L}^{c}$. Hence, $\Delta \xi=0$ in $\mathcal{L}^{c}$. $\xi$ vanishes on $\partial \Omega$ since $\phi_{i} \in H_{0}^{1}(\Omega)$. To obtain the boundary values for $\xi$ on $\partial \mathcal{L}$, we examine the eigenexpansion (5.8), which can be rearranged in the form

$$
\sum_{i \in \mathcal{S}_{+}} \alpha_{i}(0) \phi_{i}(\mathbf{x})=\phi_{0}(\mathbf{x})-\sum_{i \in \mathcal{S}_{\Pi} \cup \mathcal{S}_{0} \cup \mathcal{S}_{1}} \alpha_{i}(0) \phi_{i}(\mathbf{x}) .
$$

For $\mathbf{x} \in \partial \mathcal{L}, \phi_{i}(\mathbf{x})=0$ if $i \in \mathcal{S}_{0} \cup \mathcal{S}_{1}$ since $\phi_{i}$ for $i \in \mathcal{S}_{0}$ is supported on $\mathcal{L}^{c}$, while $\phi_{i}$ for $i \in \mathcal{S}_{1}$ is supported on $\mathcal{L}$. Consequently, for $\mathbf{x} \in \partial \mathcal{L}$, it follows from (5.7) and (5.10) that

$$
-\xi(\mathbf{x})=\sum_{i \in \mathcal{S}_{+}} \alpha_{i}(0) \phi_{i}(\mathbf{x})=\phi_{0}(\mathbf{x})-\sum_{i \in \mathcal{S}_{\Pi}} \alpha_{i}(0) \phi_{i}(\mathbf{x})=\phi_{0}(\mathbf{x})-\phi_{L} \Pi(\mathbf{x}) .
$$

Since $\Pi(\mathbf{x})=1$ for $\mathbf{x} \in \partial \mathcal{L}, \xi(\mathbf{x})=\phi_{L}-\phi_{0}(\mathbf{x})$ on $\partial \mathcal{L}$. This completes the proof of Theorem 1.1.

\section{LiMit IN ONE DIMENSION}

In this section, we focus on Theorem 1.1 in dimension $1(n=1)$ with $\Omega$ the open interval $(0,1)$ and $\mathcal{L}$ a subinterval $(a, b)$ whose closure is contained in $(0,1)$. In this case, the equations describing $\Pi$ reduce to

$$
\Pi^{\prime \prime}=0 \text { in }(0, a) \cup(b, 1), \quad \Pi=1 \text { in }[a, b], \quad \Pi(0)=\Pi(1)=0 .
$$

The solution is

$$
\Pi(x)=\left\{\begin{array}{cl}
s_{1} x & \text { if } x \in(0, a), \\
1 & \text { if } x \in[a, b], \\
s_{2}(1-x) & \text { if } x \in(b, 1),
\end{array}\right.
$$

where

$$
s_{1}=\frac{1}{a} \quad \text { and } \quad s_{2}=\frac{1}{1-b} .
$$

Hence, we have

$$
\phi_{L}=\frac{\left\langle\phi_{0}^{\prime}, \Pi^{\prime}\right\rangle_{\Omega}}{\left\langle\Pi^{\prime}, \Pi^{\prime}\right\rangle_{\Omega}}=\frac{s_{1} \phi_{0}(a)+s_{2} \phi_{0}(b)}{\frac{1}{a}+\frac{1}{1-b}}=\frac{(1-b) \phi_{0}(a)+a \phi_{0}(b)}{1-b+a} .
$$

Let us define the parameters

$$
\theta_{1}=\frac{1-b}{1-b+a} \quad \text { and } \quad \theta_{2}=\frac{a}{1-b+a} .
$$


With these definitions,

$$
\phi_{L}=\theta_{1} \phi_{0}(a)+\theta_{2} \phi_{0}(b),
$$

where $\theta_{1}>0, \theta_{2}>0$, and $\theta_{1}+\theta_{2}=1$. Thus the effect of the lightning is to make the potential $\phi_{L}$ on the lightning channel $(a, b)$ a convex combination of the potential $\phi_{0}(a)$ and $\phi_{0}(b)$ at the ends of the channel. The coefficients $\theta_{1}$ and $\theta_{2}$ in the convex combination depend on the distance between the ends of the channel and the boundary of the domain $\Omega$. It is interesting to note that the potential $\phi_{L}$ on the lightning channel only depends on the pre-flash potentials $\phi_{0}(a)$ and $\phi_{0}(b)$ at the ends of the channel; in other words, the pre-flash potential at interior points along the channel apparently has no effect on the potential that is achieved along the lightning channel after the flash. Also, notice that as one of the channel ends, say $a$ approaches the boundary, $\phi_{L}$ approaches zero since $\theta_{2}$ and $\phi_{0}(a)$ both approach 0 as $a$ approaches 0 (recall that $\left.\phi_{0}(0)=0\right)$. A more general discussion of a cloud-to-ground flash is given after Theorem 1.1.

Now let us focus on the potential change $\xi$ outside the lightning channel $\mathcal{L}$. According to Theorem 1.1,

$$
\begin{array}{lll}
\xi^{\prime \prime}=0 \text { on }(0, a), & \xi(0)=0, & \xi(a)=\phi_{L}-\phi_{0}(a), \\
\xi^{\prime \prime}=0 \text { on }(b, 1), & \xi(1)=0, & \xi(b)=\phi_{L}-\phi_{0}(b) .
\end{array}
$$

The solution is

where

$$
\xi(x)=\left\{\begin{array}{cc}
r_{1} x & \text { on }(0, a), \\
r_{2}(1-x) & \text { on }(b, 1),
\end{array}\right.
$$

Substituting for $\phi_{L}$ using (6.1), we obtain

$$
r_{1}=\frac{\phi_{L}-\phi_{0}(a)}{a} \text { and } r_{2}=\frac{\phi_{L}-\phi_{0}(b)}{1-b} .
$$

$$
r_{1}=\frac{\left(\theta_{1}-1\right) \phi_{0}(a)+\theta_{2} \phi_{0}(b)}{a}=\frac{\theta_{2}}{a}\left(\phi_{0}(b)-\phi_{0}(a)\right)=\frac{\phi_{0}(b)-\phi_{0}(a)}{1-b+a}=-r_{2} .
$$

Hence, by Theorem 1.1, we have

$$
\phi^{+}(x)=\left\{\begin{array}{cl}
\phi_{0}(x)+x \frac{\delta \phi_{0}}{1-|\mathcal{L}|} & \text { if } x \in(0, a), \\
\phi_{0}(x)-(1-x) \frac{\delta \phi_{0}}{1-|\mathcal{L}|} & \text { if } x \in(b, 1),
\end{array}\right.
$$

where $\delta \phi_{0}=\phi_{0}(b)-\phi_{0}(a)$ and $|\mathcal{L}|=b-a$ is the length of the lightning channel. Thus lightning causes a linear change in the electric potential, where the size of the linear perturbation is proportional to the pre-flash potential difference across the ends of the channel.

\section{Conclusions}

In Theorems 1.1 and 1.2, we evaluate the change in the electric potential induced by * The electric potential change in the discrete setting is evaluated by expressing the solution to (1.13) as a product of symmetric factors and analyzing the effect of $\tau$ on the eigenvalues. By (3.6), the eigenvalues grow in proportion to $\tau$, with an error term which is bounded, independent of $\tau$. The derivation of the formula for the lightning induced electric potential change in the continuous equation (1.5) is based on the properties of the eigenfunctions and eigenvalues of the generalized eigenproblem (1.15). There are four classes of eigenfunctions all of which enter into the analysis. However, the post-flash potential is represented using eigenfunctions of type 1 and type 4, functions in $H_{0}^{1}(\Omega)$ which are harmonic in both $\mathcal{L}$ and $\mathcal{L}^{c}$. The potential along the lightning channel is a constant obtained by projecting the pre-flash potential along the type 1 eigenfunction $\Pi$. Outside the lightning channel, the change in the electric potential is a linear combination of type 4 eigenfunctions, for which the associated eigenvalues are contained on the open interval $(0,1)$ with $\lambda=1 / 2$ the only possible accumulation point. 
The boundary conditions for the electric potential change outside the lightning channel are expressed in (1.10) in terms of the pre-flash potential $\phi_{0}$ and the post-flash potential $\phi_{L}$ along the lightning channel.

Acknowledgements. It was pointed out by Yaroslav Krylyuk that the analysis in [4] establishing the discrete limit (1.14) implicitly assumed that the matrices $\mathbf{A}$ and $\mathbf{B}$ in (1.13) commute. This observation led to the new analysis given in Section 3, which helped to motivate future work on the continuous equations (1.1)-(1.3). The formula (1.12) relating the change in potential to the energy of the flash was pointed out by William Koshak. We also thank the reviewers for their comments and suggestions which led to a more precise and complete paper.

\section{REFERENCES}

[1] R.A. Adams, Sobolev Spaces. Academic Press, New York (1975).

[2] B.C. Aslan, W.W. Hager and S. Moskow, A generalized eigenproblem for the Laplacian which arises in lightning. J. Math. Anal. Appl. 341 (2008) 1028-1041.

[3] R.F. Curtain and H. Zwart, An Introduction to Infinite-Dimensional Linear Systems Theory. Springer-Verlag, New York (1995).

[4] W.W. Hager, A discrete model for the lightning discharge. J. Comput. Phys. 144 (1998) 137-150.

[5] W.W. Hager, J.S. Nisbet and J.R. Kasha, The evolution and discharge of electric fields within a thunderstorm. J. Comput. Phys. 82 (1989) 193-217.

[6] W.W. Hager, J.S. Nisbet, J.R. Kasha and W.-C. Shann, Simulations of electric fields within a thunderstorm. J. Atmos. Sci. 46 (1989) 3542-3558.

[7] W.W. Hager, R.G. Sonnenfeld, B.C. Aslan, G. Lu, W.P. Winn and W.L. Boeck, Analysis of charge transport during lightning using balloon borne electric field sensors and LMA. J. Geophys. Res. 112 (2007) DOI: 10.1029/2006JD008187.

[8] C.L. Lennon, LDAR: new lightning detection and ranging system. EOS Trans. AGU 56 (1975) 991.

[9] L. Maier, C. Lennon, T. Britt and S. Schaefer, LDAR system performance and analysis, in The 6th conference on aviation weather systems, American Meteorological Society, Boston, MA (1995).

[10] T.C. Marshall and M. Stolzenburg, Voltages inside and just above thunderstorms. J. Geophys. Res. 106 (2001) $4757-4768$.

[11] B.N. Parlett, The Symmetric Eigenvalue Problem. Prentice-Hall, Englewood Cliffs, NJ (1980).

[12] D.E. Proctor, A hyperbolic system for obtaining VHF radio pictures of lightning. J. Geophys. Res. 76 (1971) 1478-1489.

[13] D.E. Proctor, VHF radio pictures of cloud flashes. J. Geophys. Res. 86 (1981) 4041-4071.

[14] V.A. Rakov and M.A. Uman, Lightning Physics and Effects. Cambridge University Press, Cambridge (2003).

[15] W. Rison, R.J. Thomas, P.R. Krehbiel, T. Hamlin and J. Harlin, A GPS-based three-dimensional lightning mapping system: Initial observations in central New Mexico. Geophys. Res. Lett. 26 (1999) 3573-3576.

[16] G. Strang, Linear Algebra and Its Applications. Thomson, Belmont, CA, 4th edn. (2006).

[17] R.J. Thomas, P.R. Krehbiel, W. Rison, T. Hamlin, J. Harlin and D. Shown, Observations of VHF source powers radiated by lightning. Geophys. Res. Lett. 28 (2001) 143-146.

[18] R.J. Thomas, P.R. Krehbiel, W. Rison, S.J. Hunyady, W.P. Winn, T. Hamlin and J. Harlin, Accuracy of the lightning mapping array. J. Geophys. Res. 109 (2004) D14207. 